\title{
BACKBENDING OF DY ISOTOPES DESCRIBED WITH THE NEUTRON-PROTON IBA PLUS TWO-QUASIPARTICLE MODEL
}

\author{
C.E. ALONSO, J.M. ARIAS and M. LOZANO \\ Departamento de Física Atómica y Nuclear, Facultad de Fisica, Universidad de Sevilla, \\ Apdo 1065. 41080 Sevilla, Spain
}

Received 18 April 1986; revised manuscript received 30 June 1986

\begin{abstract}
The formalism of the neutron-proton interacting boson model, extended to include two-quasiparticle excitations, is used in a study of the high spin states of ${ }^{154.156 .158}$ Dy isotopes. Good agreement is obtained between the calculated energies and transition rates and the experimental results.
\end{abstract}

The basic feature of the structure of the yrast line of deformed heavy nuclei can be described as an interplay between collective rotations and singleparticle excitations. For even-even nuclei the ground state band is due to the collective rotation of the nucleus around an axis perpendicular to the symmetry axis, being the moment of inertia approximately half of that corresponding to a rigid rotor because of the pairing correlations. When the angular velocity increases the Coriolis force tends to break pairs of nucleons moving in high- $j$ intruder orbits close to the Fermi surface which align their angular momenta along the rotational axis. Each of these pairs provides a part of the total angular momentum and the nucleus rotates at a lower rotational frequency. In this rotational alignment picture [1] the anomalies occurring in the yrast sequence of deformed nuclei are interpreted as being due to the intersection of the paired ground state band with a band involving one aligned quasiparticle pair (first backbending), this one with a band involving two pairs (second backbending), etc.

The most legitimate method to describe these phenomena seems to be the Hartree-Fock-Bogoliubov theory in the cranking framework $[2,3]$. From a phenomenological point of view, the decoupling model [4-7] has been extended in order to include two

\footnotetext{
Work partially supported by the Spanish Comisión Asesora de Investigación Científica y Técnica, contract number 2868-83.
}

quasiparticle states in even-even [8] and odd-odd [9] mass nuclei. Another phenomenological approach is the includion of two quasiparticle excitations in the interacting boson model [10]. This was done by Gelberg and Zemel [11] in a schematic model, which, due to the "ad hoc" nature of its hamiltonian, is not theoretically satisfactory. Using a model proposed by Yoshida et al. [12] high spin states have been described by allowing to change one of the bosons of the IBA-2 model into a pair of nucleons [13,14]. In the same approach Morrison et al. [5] have given another formulation for the inclusion of two-quasiparticle states in a boson basis, based on the philosophy of the IBFA model [16]. In the same line one can include some recent works by Faessler et al. [17].

In this paper we describe the high spin states of three Dy isotopes within the IBA plus two-quasiparticle model of Yoshida et al. [12]. It is worth noting that these Dy isotopes have been studied recently within an alternative extension of the IBA model [18]. In that model, called core-excited interacting boson mode, a nucleus is considered as a system with no constant number of bosons. The backbending phenomenon is produced by the crossing between the ground state band and another band with a larger number of bosons. The core-excited IBM overemphasizes the role of the collective degrees of freedom, thus a calculation with a more realistic model which takes into account the interplay between single-particle and collective degrees of freedom (as the one of 
ref. [12]) is desirable.

Since this model has been presented and discussed in some of the above references we summarize here its main features.

The neutron-proton IBA model yields a good description of the low-lying collective states in even-even nuclei with a set of parameters phenomenologically fitted to the experimental data. In order to describe levels of high spin and excitation energy it is assumed that one proton-boson and/or one neutron-boson is broken to form a quasiparticle pair, usually assigned to a unique parity intruder orbital with spin $j$. The total angular momentum of the pair can take on the values $J=4,6, \ldots, 2 j-1$. The couplings to angular momenta 0 and 2 are excluded in order to avoid double counting of states, because they are included through the $s$ and $d$ bosons respectively. The model space includes the IBA-2 space, i.e., states with $N_{\pi}$ proton-bosons and $N_{\nu}$ neutron-bosons, and states with $\left(N_{\pi(\nu)}-1\right)$ proton (neutron)-bosons, $N_{\nu(\pi)}$ neutron (proton)-bosons and two protons (neutrons). The mixing between states with different number of bosons is introduced through a bosonnumber-changing term in the quadrupole operator:

$$
\begin{gathered}
Q_{\rho}=Q_{\rho}^{\mathrm{B}}+\alpha_{\rho}\left(a_{j}^{\dagger} \tilde{a}_{j}\right)^{(2)}+\beta_{\rho}\left[\left(a_{j}^{\dagger} a_{j}^{\dagger}\right)^{(4)} \tilde{d}_{\rho]}(2)\right. \\
-\beta_{\rho}\left[d_{\rho}^{\dagger}\left(\tilde{a}_{j} \tilde{a}_{j}\right)^{(4)}\right]^{(2)}, \quad \rho=\nu, \pi
\end{gathered}
$$

where

$Q_{p}^{\mathrm{B}}=\left(d_{p}^{\dagger} \tilde{s}_{p}+s_{p}^{\dagger} \tilde{d}_{p}\right)^{(2)}+\chi_{\rho}\left(d_{p}^{\dagger} \partial_{p}\right)^{(2)}$,

with $a_{j}^{\dagger}=a_{(j m)}^{\dagger}$ being the nucleon creation operator $\left(\tilde{a}_{(j m)}=(-1)^{j-m} a_{(j-m)}\right)$ and, $s^{\dagger}$ and $d^{\dagger}$ the $s$ and $\mathrm{d}$ boson creation operators.

The model hamiltonian is

$$
H=H_{\mathrm{B}}+H_{\mathrm{F}}+V_{\mathrm{BF}},
$$

where $H_{\mathrm{B}}$ is the IBA-2 hamiltonian [19] and $H_{\mathrm{F}}$ the fermion hamiltonian that, restricted to a single $j$-shell, takes the form

$$
H_{\mathrm{F}}=\sum_{m} E_{j} a_{j m}^{\dagger} a_{j m}+\frac{1}{2} \sum_{J, M} V^{J}\left(a_{j}^{\dagger} a_{j}^{\dagger}\right)^{J M}\left(\tilde{a}_{j} \tilde{a}_{j}\right)^{J M} .
$$

For the interaction between bosons and fermions, $V_{\mathrm{BF}}$, a quadrupole-quadrupole interaction is assumed:

$$
V_{\mathrm{BF}}=\kappa^{\prime}\left(Q_{\pi} Q_{\nu}-Q_{\pi}^{\mathrm{B}} \cdot Q_{\nu}^{B}\right),
$$

where $Q_{\nu}$ is the generalized quadrupole operator given by eq. (1), and $Q_{\rho}^{\mathrm{B}}$ is the boson quadrupole operator of eq. (2).

The Dy isotopes we are intersted in have $Z=66$ and $88 \leqslant N \leqslant 92$. Thus, valence protons are the $50-82$ major shell and valence neutrons in the 82-126 major shell. The orbitals with larger angular momentum in these shells are $1 \pi \mathrm{h}_{11 / 2}$ and $1 \nu \mathrm{i}_{13 / 2}$ for protons and neutrons respectively. In addition to these ones the $1 \nu \mathrm{h}_{9 / 2}$ orbital is very close to the Fermi surface in all the isotopes studied and it may be important in the analysis of the backbending phenomena in these nuclei. Because of that, we have included in our study both orbits for neutrons: $1 \nu \mathrm{i}_{13 / 2}$ and $1 \nu \mathrm{h}_{9 / 2}$ and the $1 \pi \mathrm{h}_{1 / / 2}$ for protons.

First of all, a description of the even-even corenuclei in terms of the IBA-2 model is needed. For instance, the study of the ${ }^{154} \mathrm{Dy}$ implies the IBA-2 description of ${ }^{154} \mathrm{Dy},{ }^{152} \mathrm{Dy}$ and ${ }^{152} \mathrm{Gd}$, since the model space is given in this case by,

$$
\begin{gathered}
\left.\left.\right|^{154} \mathrm{Dy}\right\rangle \otimes \mid{ }^{152} \mathrm{Dy}+2 \text { neutrons }> \\
\left.\otimes \mid{ }^{152} \mathrm{Gd}+2 \text { protons }\right\rangle .
\end{gathered}
$$

In table 1, the IBA-2 parameters used in this study for the description of the even-even nuclei implied are displayed. For ${ }_{66} \mathrm{Dy}$ isotopes they have been obtained by extrapolating those given in ref. [19] for ${ }_{60} \mathrm{Nd},{ }_{62} \mathrm{Sm}$ and ${ }_{64} \mathrm{Gd}$. For isotopes they have been taken from the same reference and slightly readjusted in order to improve the description of the lowest states. For the two-body interaction between fermions (in $1 \nu \mathrm{h}_{9 / 2}, 1 \nu \mathrm{i}_{13 / 2}$ and $1 \pi \mathrm{h}_{11 / 2}$ ) a surface delta interaction (SDI) has been used with strength adjusted to lower the $J=0$ state by $2 \mathrm{MeV}$. The single-particle energies obtained as a result of fitting (fig. 1) coincide approximately with the quasiparticle energies obtained from a standard BCS calculation for the orbitals of interest. The additional parameters, in $V_{\mathrm{BF}}$ (eq. (5)), have been taken constant for the three isotopes: $\alpha_{\pi}=\alpha_{\nu}=-0.4$ and $\beta_{\nu}=\beta_{\nu}=0.5$, or varying smoothly from isotope to isotope: $\kappa^{\prime}=0.04$, $0.05,0.06 \mathrm{MeV}$ for $N=88,90,92$ respectively.

For the ${ }_{66} \mathrm{Dy}$ isotopes there is also experimental information on $B(\mathrm{E} 2)$ 's. The study of these values will give us a good test of the model wave functions. The electric quadrupole operator is written as

$$
\begin{aligned}
& T^{(\mathrm{E} 2)}=\sum_{\rho=\pi . \nu}\left\{e_{\rho}^{\mathrm{B}} Q_{\rho}^{\mathrm{B}}+e_{\rho}^{\mathrm{F}} \alpha_{\rho}\left(\alpha_{j}^{\dagger} \tilde{a}_{j}\right)^{(2)}\right. \\
& \left.+\beta_{\rho} e_{\rho}^{\mathrm{B}}\left[\left(a_{j}^{\dagger} a_{j}^{\dagger}\right)^{(4)} \tilde{d}_{\rho}\right]^{(2)}-\beta_{\rho} e_{\rho}^{\mathrm{B}}\left[d_{\rho}^{\dagger}\left(\tilde{a}_{j} \tilde{a}_{j}\right)^{(4)}\right]^{(2)}\right\},
\end{aligned}
$$

where $e_{\pi}^{\mathrm{B}}\left(e_{\nu}^{\mathrm{B}}\right)$ is the proton-boson (neutron-boson) effective charge and $e_{\pi}^{\mathrm{F}}\left(e_{\nu}^{\mathrm{F}}\right)$ is proportional to the 
Table 1

Parameters $\epsilon, \kappa$ in the IBA-2 hamiltonian (in MeV). All the other parameters in the IBA-2 hamiltonian are taken the same as in ref. [19]

\begin{tabular}{|c|c|c|c|c|c|}
\hline \multirow[t]{2}{*}{ Nucleus } & \multirow[t]{2}{*}{ Parameter } & \multicolumn{4}{|l|}{$N$} \\
\hline & & 86 & 88 & 90 & 92 \\
\hline \multirow[t]{2}{*}{${ }_{60} \mathrm{Dy}$} & $\epsilon$ & 0.717 & 0.620 & 0.592 & 0.550 \\
\hline & $\kappa$ & -0.028 & -0.055 & -0.073 & -0.070 \\
\hline \multirow[t]{2}{*}{${ }_{0.4} \mathrm{Gd}$} & $\epsilon$ & & 0.596 & 0.550 & 0.460 \\
\hline & $\kappa$ & & -0.051 & -0.080 & -0.081 \\
\hline
\end{tabular}

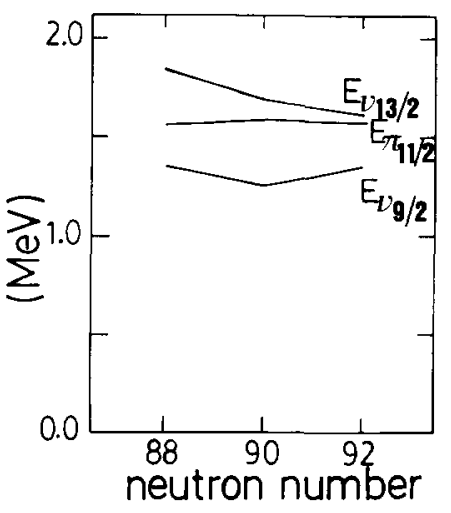

Fig. 1. Quasiparticle energies used in $H_{\mathrm{F}}$ for the single-particle orbits included in this study.

proton (neutron) effective charge.

The boson effective charges in the $T^{\mathrm{E} 2)}$ operator have been determined by adjusting the experimental value of $B\left(\mathrm{E} 2 ; 2_{1}^{+} \rightarrow 0_{1}^{+}\right)$for each isotope. For the charges $e_{\pi}^{\mathrm{F}}$ and $e_{\nu}^{\mathrm{F}}$ in eq. (6) we have used $e_{\pi}^{\mathrm{F}}=0.53 e$ $\mathrm{b}$ and $e_{\nu}^{\mathrm{F}}=0.21 e \mathrm{~b}$. These values have been obtained by using harmonic oscillator radial wave functions and proton (neutron) effective charge equal to $1.5 \mathrm{e}$ $(0.5 e)$.

The level structure of the ${ }^{154} \mathrm{Dy}$ isotope suggests a small prolate deformation [20] although there are predictions of a prolate-to-oblate transition above spin $40 \hbar$ [21,22]. In fig. $2 a$ we present the results of the calculations in comparison with the experimental levels of Pakkanen et al. [20]. The configuration responsible for the backbending in this case in our calculation is ${ }^{152} \mathrm{Gd} \otimes\left(\pi \mathrm{h}_{11 / 2}\right)^{2}$, being that of two quasineutrons considerably higher in energy. Our study yields a $g$-factor of the states around the backbending higher than the collective $g$-factor due to the contribution of the two protons. The transition probabilities $B(\mathrm{E} 2)$ calculated are presented in fig. 3a along with the experimental data [20].

The backbending in ${ }^{156} \mathrm{Dy}$ is commonly interpreted as the transition from the ground state rotational band to the aligned two-quasiparticle $i_{13 / 2}$ neutron band [23]. Fig. 2b shows the result of the study done using IBA-2 plus two quasiparticles. The configurations including two-quasineutrons are lower in energy. It is important to notice that both configurations ${ }^{154} \mathrm{Dy} \otimes\left(1 \nu \mathrm{h}_{9 / 2}\right)^{2}$ and ${ }^{154} \mathrm{Dy} \otimes\left(1 \nu \mathrm{i}_{13 / 2}\right)^{2}$ are competitive at the point of the first backbending, being that of two neutrons $1 h_{9 / 2}$ a bit lower in energy. Theoretical and experimental [24] $B(E 2)$ values are shown in fig. $3 \mathrm{~b}$. Our calculation follows nicely the sudden fall for $I=16 \hbar$ and the rising for $I=18 \hbar$. The interpretation of the third experimental point in fig. $3 \mathrm{~b}(I=6 \hbar)$ is difficult in the framework of the interplay between two rotational bands, since the excitation energy of the first $6^{+}$level is only $770 \mathrm{keV}$. As can be seen in fig. $3 b$ we are not able to reproduce such a point. In our study an additional band crossing between the band with two quasineutrons and that of two quasiprotons is observed at spin $24 \hbar$. This band crossing is not seen in the plot of $E(I)-E(I-2)$, but it is clear in the behavior of the $B(\mathrm{E} 2)$. The experimental data for $B(E 2)$ seem to indicate such a lowering at $I=24 \hbar$. In our calculation, in the crossing point, the bands have almost no mixing between them and therefore the jump is too exaggerated. A small amount of mixing would contribute to improve the agreement. This result is in agreement to one measurement of $g$-factors for this isotope [25]. In this experiment there is a clear negative contribution to the collective $g$-factor at $I \simeq 14 \hbar$ which is due to the alignment of two quasineutrons and a positive con- 


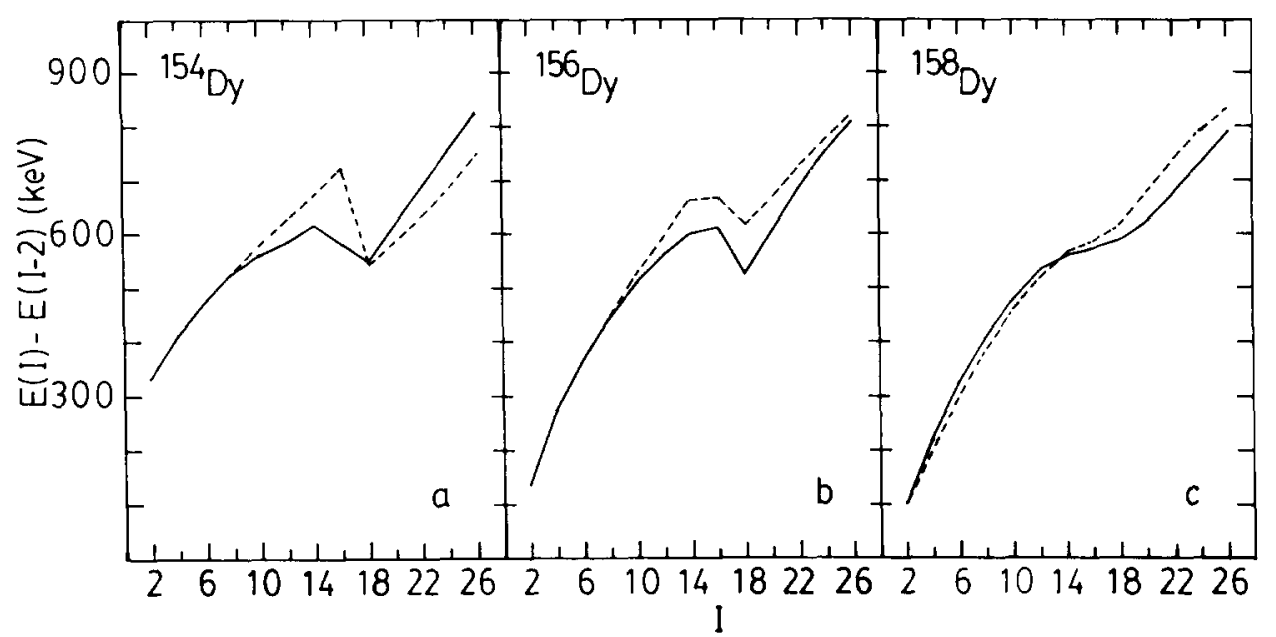

Fig. 2. Comparison of the experimental energy differences in the yrast line (full line) with the results of the present study (dashed line) for: (a) ${ }^{154} \mathrm{Dy}$, (b) ${ }^{156} \mathrm{Dy}$ and (c) ${ }^{15 x} \mathrm{Dy}$.

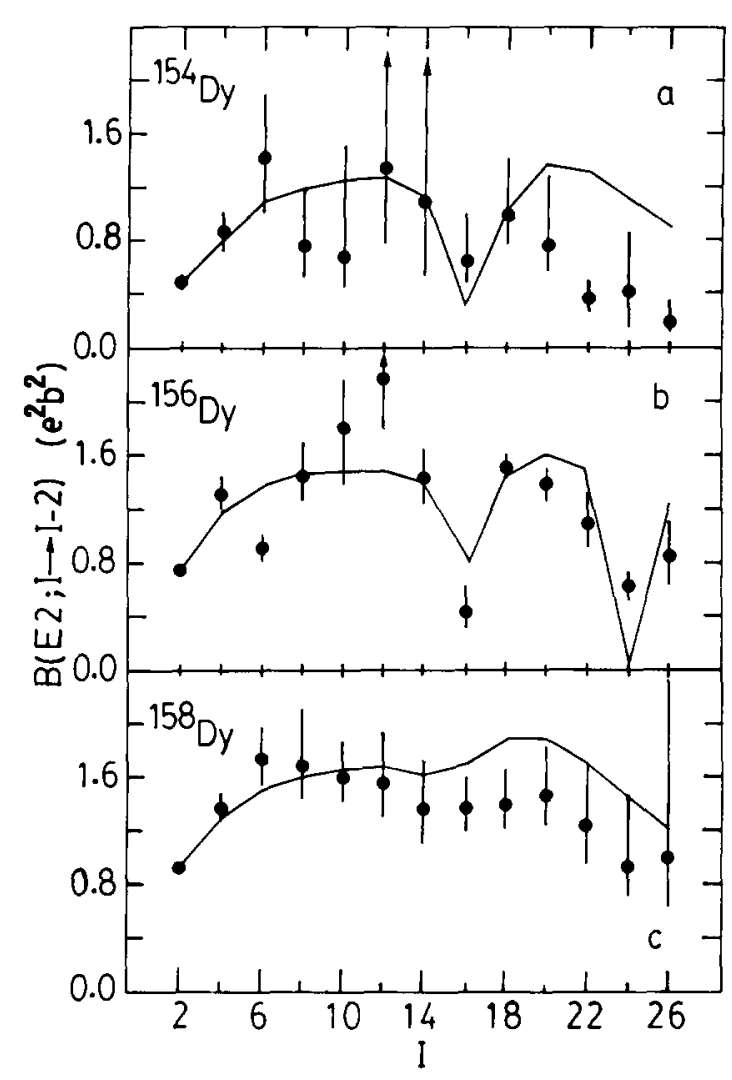

Fig. 3. Comparison of the experimental $B(E 2)$ values for transitions in the yrast line (points) with the results of the present study (full line) for: (a) ${ }^{154} \mathrm{Dy}$, (b) ${ }^{156} \mathrm{Dy}$ and (c) ${ }^{158} \mathrm{Dy}$. tribution to it at $I \simeq 24 \hbar$ due to the alignment of a couple of quasiprotons. It is important to note that this experiment is not conclusive about the backbending nature since the (HI, $x \mathrm{n})$ reaction employed populates states on and above the yrast line. Because of that, a new measurement of $g$-factors is desirable in order to clarify if there is an additional band crossing at around $I=24 \hbar$, as the experiment of ref. [25] and our calculation indicate.

The calculated and experimental [26,27] energy differences and $B(E 2)$ values for ${ }^{158}$ Dy are presented in figs. $2 \mathrm{c}$ and $3 \mathrm{c}$. In the case of this isotope the configuration responsible for the backbending is known, since a measurement of the average $g$-factors in this region exists [26] and demonstrates that the alignment of an $i_{13 / 2}$-neutron pair is the dominant effect in the intrinsic structure of the yrast band around the backbending region. In our calculation the configurations with two quasineutrons are considerably lower than that with two quasiprotons, being the responsible for the first backbending, in agreement with the results of ref. [27]. In this case the configuration with two neutrons $l_{13 / 2}$ is the most important in the backbending region, due basically to the behavior of the quasiparticle energies (see fig. 1). Because of that, this configuration is expected to be the basic one for the study of heavier isotopes. In fig. $3 \mathrm{c}$ the $B(\mathrm{E} 2)$ values for this isotope are displayed along with the experimental data $[26,27]$. The basic 
behavior of $E(I)-E(I-2)$ and $B(\mathrm{E} 2)$ versus $I$ is well reproduced.

Some data on side bands are also known for these isotopes. Their behavior is reasonably well reproduced in our study. The analysis of these bands together with a comparison between our results and those of ref. [18] will be presented in detail in a forthcoming paper.

In conclusion, we show that the IBA-2 plus two quasiparticle model is succesful in reproducing the main features of the energy spectra and electric quadrupole transition rates in the region of the first backbending phenomenon in the transitional isotopes ${ }^{154} \mathrm{Dy},{ }^{156} \mathrm{Dy}$ and ${ }^{158} \mathrm{Dy}$. It is also a result of the present analysis that the responsible for the backbending in these nuclei is a two-proton quasiparticle configuration for ${ }^{154} \mathrm{Dy}$, and the alignment of two quasineutrons for ${ }^{156.158} \mathrm{Dy}$. In ${ }^{156} \mathrm{Dy}$ an additional band crossing with a two-proton configuration at spin $24 h$ is deduced from the present analysis. Experimental measurements of $g$-factors for ${ }^{154.156} \mathrm{Dy}$ are highly desirable in order to know the nature of the yrast line at high spin in these nuclei.

The authors acknowledge helpful discussions with F. Iachello.

\section{References}

[1] R.M. Lieder and H. Ryde, Advances in nuclear physics, Vol.10, M. Baranger and E. Vogt (Plenum, New York, 1978) p. 1 .

[2] H.J. Mang, Phys. Rep. 18 (1975) 325.

[3] M. Diebel, A.N. Mantri and U. Mosel, Nucl. Phys. A 345 (1980) 72.

[4] F.S. Stephens, R.M. Diamond, J.R. Leigh, T. Kammuri and K. Nakai, Phys. Rev. Lett. 29 (1972) 438.

[5] J. Meyer ter Vehn, Nucl. Phys. A 249 (1975) 111, 141.

[6] H. Toki and A. Faessler, Nucl. Phys. A. 253 (1975) 231.

[7] A. Faessler, Proc. Gull Lake Conf. on Nuclear spectroscopy (Gull Lake, MI, 1979), Lecture Notes in Physics, Vol. 119 (Springer, Berlin, 1980).
[8] H.L. Yadav, H. Toki and A. Faessler, Phys. Rev. Lett. 39 (1977) 1128;

H.L. Yadav, A. Faessler, H. Toki and B. Castel, Phys. Lett. B 89 (1980) 3.

[9] H. Toki, H.L. Yadav and A. Faessler, Phys. Lett. B 81 (1979) 119.

[10] A. Arima and F. Iachello, Ann. Phys. (NY) 99 (1976) 253: 111 (1978) 201; Phys. Rev. Lett. 40 (1978) 385.

[11] A. Gelberg and A. Zemel, Phys. Rev. C22 (1980) 937.

[12] N. Yoshida, A. Arima and T. Otsuka, Phys. Lett. B 114 (1982) 86

[13] H. Hanewinkel, W. Gast, U. Kaup, H. Harter, A. Dewald. A. Gelberg, R. Reinhardt, P. von Brentano. A. Zemel, C.E. Alonso and J.M. Arias, Phys. Lett. B 133 (1983) 9.

[14] N. Yoshida and A. Arima, Phys. Lett. B 164 (1985) 231.

[15] I. Morrison, A. Faessler and C. Lima, Nucl. Phys. A 372 (1981) 13 .

[16] F. Iachello and O. Scholten, Phys. Rev. Lett., 43 (1979) 679.

[17] A. Faessler, Proc. Intern. Summer School La Rábida 1982, Lecture Noptes in Physics, Vol. 168, eds. G. Madurga and M. Lozano (Springer, Berlin, 1982) p. 267;

A. Faessler, S. Kuyucak, A. Petrovichi and L. Petersen, Nucl. Phys. A 438 (1985) 78.

[18] K. Heyde, J. Jolie, P. van Isacker and M. Waroquier, Phys. Rev. C 29 (1984) 1428.

[19] O. Scholten, Ph.D. thesis, Groningen University (1980).

[20] A. Pakkanen, Y.H. Chung, P.J. Daly, S.R. Faber, H. Helppi, J. Wilson, P. Chowdhury, T.L. Khoo, I. Ahmad, J. Borggreen, Z.W. Grabowski and D.C. Radford, Phys. Rev. Lett. 48 (1982) 1530.

[21] S. Aberg, in: Elementary modes of excitation in nuclei, eds. A. Bohr and R.A. Broglia (North-Holland, Amsterdam, 1977).

[22] C.G. Anderson et al., Phys. Scr. 24 (1981) 266.

[23] H. Emling, Proc. XIV Masurian Summer School on Nuclear physics (Mikolajki, 1981).

[24] H. Emling, E. Grosse, R. Kulessa, D. Schwalm and H.J. Wollersheim, Nucl. Phys. A 419 (1984) 187.

[25] N. Rud, D. Ward, H.R. Andrews, O. Hausser, P. Taras, J. Keinonen, N. Neiman, R.M. Diamond and F. Stephens, Phys. Lett. B 101 (1981) 35.

[26] H. Emling, E. Grosse, D. Schwalm, R.S. Simon, H.J. Wollersheim, D. Husar and D. Pelte, Phys. Lett. B 98 (1981) 169.

[27] G. Seiler-Clark, D. Pelte, H. Emling, A. Balanda, H. Grein, E. Grosse, R. Kulessa, D. Schwalm, H.J. Wollersheim, M. Hass, G.J. Kumbartzki and K.H. Speidel, Nucl. Phys. A 399 (1983) 211 . 